\title{
Exploring the Effect of Prior Knowledge and Gender on Undergraduate Students' Knowledge Structures in Chemistry
}

\author{
Ozcan Gulacar ${ }^{1 *}$, Alexandra Milkey ${ }^{1}$, Spivey McLane ${ }^{1}$ \\ ${ }^{1}$ University of California, Davis, California, USA
}

Received 19 June 2018 - Revised 15 December 2018 • Accepted 11 January 2019

\begin{abstract}
Success in chemistry requires not only the ability to recruit prior knowledge but also the ability to establish strong connections between new and existing concepts to form knowledge clusters around core principles. How these knowledge structures are organized can be used to understand the relationships between concepts within a student's mind. 618 undergraduate students in a general chemistry course participated in this study at a US institution. The purpose of this study was to determine the effect, if any, that prior knowledge in chemistry and mathematics and gender have on the formation of students' knowledge structures. In addition, the structures were analyzed to identify the hidden connections between macroscopic, submicroscopic, and symbolic representations of chemical knowledge. To visualize these structures, a word association test (WAT) was created to determine concept relatedness. Student response data was then transformed into a series of distances by a computer program called JPathfinder, which created visual representations of the knowledge structures in the Gephi platform. The meaning and implications of these structures were discussed to provide ideas for teaching interventions that focus on weakly associated basic general chemistry concepts. The potential uses of WAT were also shared to help educators identify student misconceptions.
\end{abstract}

Keywords: assessment, prior knowledge, gender, chemistry triplet, introductory chemistry, knowledge structures, problem-solving

\section{INTRODUCTION}

The constructivist theory of learning has changed educators' and researchers' focus from teaching to learning (Forster, 1999; Von Glasersfeld, 1990). The traditional question, "How should we teach?" has been replaced with an entirely different but very relevant question, "How do people learn?". This shift in emphasis resulted in new teaching methods that put students at the center of instruction (Baker, 1993). The central idea of constructivism is that learning is an active process in which students interact with the material and build connections with what they already know or believe to be true (Ausubel, Novak, \& Hanesian, 1986). Although contradicting explanations exist among constructivists' theories, all emphasize pre-existing knowledge's role in creating new connections within the knowledge structure, which is the interconnected network of information in a learner's mind (Noss \& Hoyles, 2017).

Chemistry is intrinsically difficult for most undergraduate students, as it demands constant attention to the intersectionality of concepts within the course (Gulacar \& Bowman, 2014). Johnstone (1982) identifies three levels of representation - macroscopic, submicroscopic, and symbolic - that one must use to solve chemical problems. All together, these are often referred to as the chemistry triplet and have become a foundation in chemistry and chemistry learning (Taber, 2013; Talanquer, 2011). Although some disparity exists about what each corner of the triplet stands for - be it levels of understanding, different representations of matter and the changes, separate lenses through which one view chemistry, or something else entirely - together they provide an adequate model that can explain much of the difficulty students have in learning chemistry.

(C) 2019 by the authors; licensee Modestum Ltd., UK. This article is an open access article distributed under the terms and conditions of the Creative Commons Attribution License (http://creativecommons.org/licenses/by/4.0/). \ogulacar@ucdavis.edu (*Correspondence) \aamilkey@ucdavis.edu \sdmclane@ucdavis.edu 


\section{Contribution of this paper to the literature}

- Previous studies involving knowledge structures have focused on smaller regions of students' mind maps involving targeted concepts. This study aimed to represent all concepts in one figure for each student group to facilitate comparisons between groups.

- Methods included multiple layers of calculation and visualization techniques to obtain the most accurate models of students' knowledge base. After processing the raw data, Relatedness Coefficients were calculated and entered into JPathfinder to determine spatial distances, which were then transferred to Gephi to generate $3 \mathrm{D}$ structures.

- The study investigated the correlation between several variables such as gender, mathematics and chemistry placement scores, and changing knowledge structures.

The vast majority of this difficulty comes in making the connection between the three levels, especially the macroscopic and submicroscopic (Hoffmann \& Laszlo, 1991; Johnstone, 2006). Bridging these gaps requires that students have careful observations of changes at the macroscopic level as well as the abilities to interpret those changes and explain what happens at the atomic level. For example, students may understand that chemical substances can be mixed together to produce a chemical change, the notation used to denote and balance chemical equations, and that individual particles are what carry out the reaction; however, it may be difficult to link these images. Failing at this stage might cause a lack of ability to solve the chemistry problems requiring the intersection of macroscopic observation and submicroscopic interpretation of the phenomena (Pinarbasi \& Canpolat, 2003). Hence, one of the goals in this study was to examine if the chemistry triplet had any basis in the way students organized their knowledge of general chemistry. Further discussions of the triplet will take place in the Results and Discussion section.

An effective knowledge of chemistry must include strong connections between scientific, mathematical, and logical concepts (Bird, 2010; Kempa \& Nicholls, 1983). Without an understanding of fundamental concepts and the ability to connect often-abstract ideas and symbols to what happens in the visible world, students cannot effectively apply these ideas in the lab or in conceptual analysis. Exploring the nature of these connections can help educators who seek out new methods to pinpoint where learning or processing deficiencies occur as well as to examine different predictive factors to determine students' success in chemistry.

Among many factors, placement exams are often used to analyze students' understanding and predict their success in a discipline. Many universities now require a passing score on entry level mathematics and chemistry placement exams before students can enroll in general chemistry courses. Mathematical ability has been long recognized as one of the important requirements for being successful in general chemistry (Rixse \& Pickering, 1985). Recent studies suggest that mathematics placement exams tend to show a significant correlation between scores earned on the test and final grades earned in chemistry courses while also highlighting trouble areas and concepts for students (Kilner, 2014). Universities consider such exams to be significant indicators of students' success and implement them to measure incoming students' understanding of these fundamental subject areas and to ensure that they are prepared for their first course load. The chemistry placement exam for the university where the study took place asks questions involving algorithmic and conceptual knowledge of chemistry as well as pure "mathematics problems" such as solving algebraic equations, revealing that the university emphasizes a fundamental understanding of mathematics as readiness for enrolling in a general chemistry course. The goal of this study is to examine the presence of a correlation between this existing knowledge and the development of connections made between newly-learned concepts in the first course in the general chemistry series.

Another approach to understanding the variation in problem-solving processes is to look at analytical functioning of the brains of students of each gender and how such processing may correlate to performance on the mathematics and chemistry placement exams. Speck, et al. (Speck et al., 2000) analyzed various aspects of the thinking process from this viewpoint and found regions of the brain that correlate to word processing. In their findings, men exhibited left-lateralized activation, whereas women demonstrated bilateral activation upon performing working memory tasks (Speck et al., 2000). Left-lateralized thinking is commonly associated with analytical and logical performance, whereas bilateral thinking utilizes both hemispheres of the brain to promote organizational learning. In addition, the performance of both genders on standardized tests has been analyzed to find inherent differences between the problem-solving and thinking patterns of the genders (Shibley Jr, Milakofsky, Bender, \& Patterson, 2003). Both studies illuminated considerable differences in the physical processing and performance between genders, but neither provided a comprehensive understanding of the individuals' cognitive processes and where missteps in understanding may occur. Thus, differences that arise in the comparison of genders remain of great interest to scientific researchers.

Students' understanding of targeted topics and concepts can be evaluated through critical analysis of their knowledge structures that are constructed based on the integration of new information into previously-known 
material. Prior knowledge, relatedness of concepts, and computational skills are factors that affect the knowledge structures that students create (Bodner, 1986; Lee, 1985; Lee \& Fensham, 1996; Lee, Goh, Chia, \& Chin, 1996). As concepts get connected to the previous ones closely in the mind of learner and fundamental concepts take a more centralized role in the knowledge structure, student understanding of course material is expected to solidify and their success in a class is predicted to increase. Word Association Tests (WAT) are one way of identifying knowledge structures constructed in the mind of learners (Clauser, 2007; Jung, 1910). A WAT is based on the principle that when given a stimulus, such as a word, individuals will automatically connect the idea to a variety of other concepts that they deem to be related. These related concepts reveal how responders think of each stimulus. WAT has been used in many different fields with the aim of reaching a variety of goals; the purpose of the WAT administered here was to visualize associations between concepts by assigning broad concepts that formed the basis of the general chemistry course as stimuli and looking for similar responses between them (Kostova, 2008). The WAT can take many different forms depending on how many times each stimulus is listed and how many responses participants are asked to give (Kostova, 2008). WATs have been used to examine the knowledge structures of students throughout a variety of disciplines (Kurt, Ekici, Aktas, \& Aksu, 2013; Yücel \& Özkan, 2015) and have proven to be a viable tool in the understanding of knowledge structures of different student groups.

Nonetheless, it is important to keep in mind that neither anatomical analyses nor WAT results alone can provide a complete representation of the thinking processes that occur within an individual's mind. The advantage of WATbased data collection in analyzing knowledge structures is that subjects are not predisposed to any restraints on their thinking because the measurement is based on spontaneous associations, which reduces the amount of bias on the data (Wagner, Valencia, \& Elejabarrieta, 1996).

The study was guided by the following research questions:

1. How does students' prior knowledge in mathematics and chemistry influence their knowledge structures in the first course in a general chemistry series?

2. Does gender influence the way students organize their knowledge in a general chemistry course?

3. What do students' knowledge structures reveal about their understanding of the particle nature of matter?

The study also aimed to interpret the structures determined through WAT in order to suggest relevant and effective teaching strategies in chemistry to encourage metacognition among students and make them aware how the important content stored in their memory are categorized and associated, forming clusters of relevant concepts.

\section{METHOD}

In order to address the research questions, a large amount of data was collected and analyzed. The details of the study, including its design and participants, are shared in the following paragraphs.

\section{Participants}

The participants in the study were drawn from students enrolled in a general chemistry course at a research university located in northeastern California during the winter of 2016. The course is typically taken by first-year and third-year-transfer undergraduates, most of whom are between the ages of eighteen and twenty-one. The course was available to any student who wished to take it, provided they passed two placement tests in chemistry and mathematics.

All students in the course were given a link to an optional online survey. Out of 1,201 students who were invited to participate, 618 elected to do so. Participants were compensated with extra credit. The detailed information for the participants is provided in Table 1.

Table 1. Participant Demographics

\begin{tabular}{lc}
\hline Category & Number of Students (Percentage) \\
\hline STEM Major / Non-STEM Major & $439(71.2 \%) / 199(29.8 \%)$ \\
\hline Female / Male & $401(65 \%) / 216(35 \%)$ \\
\hline Took AP Chemistry / Did not Take AP Chemistry & $102(16.6 \%) / 516(83.4 \%)$ \\
\hline Passed Chemistry Placement Test (CPT) / Did not Pass CPT & $346(58.2 \%) / 272(41.8 \%)$ \\
\hline Passed Mathematics Placement Test (MPT) / Did not pass MPT & $522(89.2 \%) / 101(10.8 \%)$ \\
\hline
\end{tabular}

Both placement tests were university requirements for students to complete before they could enroll in the course. It was required that a student received a minimum score of 24 out of 44 total questions on the chemistry placement test and a minimum score of 30 out of 60 total questions on the mathematics placement test to enroll in the general chemistry class. Students who did not pass the chemistry placement test were required to complete a workload chemistry course intended to refresh their memories on fundamental chemistry concepts before they could enroll. 
The high- and low- scoring sets of students were determined using these placement tests that were multiplechoice and administered online during the summer of 2015. Because of the significant percentage of students who passed the mathematics placement test $(89.2 \%)$ and the resulting inequality in sample sizes, it was decided that simply grouping the students by who had passed each test and who had not would not be a strong enough indicator of background knowledge. When one WAT population is small, extraneous answers are given more weight and can influence the overall responses to a greater degree. To avoid this, the scores were sorted numerically, and the 200 highest scores for each subject test were used as the high- scoring students while the 200 lowest scores for each were used as the low-scoring. For chemistry, all of the low-scoring students did not pass the placement test and were placed in a workload chemistry class before they could enroll in general chemistry.

Gender was not taken into consideration when grouping the students; however, after the high- and lowachieving groups had been selected, they were analyzed to find the gender breakdowns of each. The genders of each set of students were as follows: 142 students (71\%) of the top students in mathematics were female, and 58 students (29\%) were male. Of the low- scoring students in mathematics, $117(58.5 \%)$ were female and $83(41.5 \%)$ were male. In chemistry, females made up $72 \%$ (144 students), and males $28 \%$ (56 students) of the high- scoring students. Of the low- scoring chemistry students, 107 (53.5\%) were female and $93(46.5 \%)$ were male. For comparative purposes, the overall participant demographics were $65 \%$ female and $35 \%$ male.

\section{Instrumentation}

To develop the Word Association Test (WAT), the following nine stimulus words were chosen by two chemistry professors and three chemistry and science education graduate students: atom, bonding, energy, matter, change, forces, stoichiometry, structure, and reaction. These words were selected based on their importance in the first course of this general chemistry series and are not meant to be representative of the entirety of general chemistry. Instead, they were intended to measure students' overall understanding of this specific course and how effective professors were at teaching these concepts, which were the only chemistry themes that a student was expected to know after finishing the course. For this class, the textbook used was the 10 th edition of General Chemistry: Principles and Modern Applications (Petrucci, Herring, Madura, \& Bissonnette, 2011). The stimuli are significant to chemistry and as such are integral in other general chemistry classes (Murphy, Holme, Zenisky, Caruthers, \& Knaus, 2012). However, as stated above, they are not intended to be representative of general chemistry entirely.

The words were made into a WAT using Qualtrics, an online program intended for data collection and analysis. The students were asked to complete the WAT by responding to each stimulus with the five words that they first thought of when they read each stimulus. They had 45 seconds per stimulus to do so. The time constraint was used to gather more accurate data about the connection between stimulus and response words (Bahar \& Hansell, 2000; Gulacar, Sinan, Bowman, \& Yildirim, 2015; Nakiboglu, 2008). Limiting response time allows a for a less biased response to the information, as with more time students might change their answers based on what they believe is the desired response (Bahar, Johnstone, \& Sutcliffe, 1999; Shavelson, 1972). Therefore, the responses given are derived from a participant's cognitive process that formulates linkages among the responses and their relationship to the larger theme, the stimulus word. Some researchers argue that the order in which students give their responses is indicative of how closely related they are within the students' knowledge structure (Bahar et al., 1999). This hierarchical relationship between words (Bahar et al., 1999) suggests that semantically similar words are accessed more quickly in an individual's knowledge structure and that concepts that are more removed from immediate association are not accessed if a time constraint is put into place (Ashcraft, 1978; Kounios \& Holcomb, 1992).

\section{Data Analysis}

Each response word within the dataset was examined and fitted into an overarching idea that was deemed a code word. One example includes the responses carbon, hydrogen, and oxygen; all were fit under the code "element", as it was believed that students were thinking primarily of the concept of "element" when they gave the response. Overarching codes for complex responses were brought to consensus in group meetings that consisted of a professor and several undergraduates. Codes were chosen based on common background ideas without losing any pertinent information. This method was used to both organize the data as well as remove answers unrelated to chemistry.

The frequencies used to determine the top twenty-five response words for each stimulus were calculated according to the hypothesis that students' first responses were the concepts they most closely associated with each stimulus. For a given response word, the frequency for each stimulus was calculated as follows:

Overall frequency $=1.0 * F_{1}+0.8 F_{2}+0.6 F_{3}+0.4 F_{4}+0.2 F_{5}$

where $F_{1}$ is the total number of times it appeared as a first response, $F_{2}$ is the number of times it appeared as a second response, and so on. Words were then ranked by overall frequency to determine the top twenty-five responses to each stimulus word. 


\section{Numerical Interpretation}

The lists of the top twenty-five responses were used to calculate relatedness coefficients that indicate the strength of the connection between stimuli. A formula developed by Garskof and Houston (1963) was implemented. One modification was made: the top twenty-five responses included each stimulus as the first response, so each list was comprised of each stimulus followed by the top twenty-four responses. This modification not only allowed connections to be made between stimuli when they appeared as responses to other stimuli but also aided in the identification of overlap between stimuli.

The relatedness coefficients were inputted into JPathfinder, a program that generates networks based on proximities such as similarity and distance. The relatedness coefficients for each data category were used as similarity values to construct upper triangular matrices that bounded the stimulus words, represented as nodes in the knowledge structures, to establish links within the network. JPathfinder converted the relatedness coefficients into distances based on a formula developed by Schvaneveldt (1990) that determined how close or far apart the nodes would be in a student's knowledge structure. The higher the value of the relatedness coefficient, the shorter the distance is between nodes and subsequently, the more associated the concepts in a student's mind. These distance values were later utilized for visualization of the knowledge structure in the Gephi program. JPathfinder also recognized the central concept for each network. Central concepts for each structure were based on a calculation of eccentricity in an initial Pathfinder network; the smaller the deviation from the standard orbital, the more central the concept is to the rest of the structure. Larger values of eccentricity denote a greater deviation from the center.

\section{Visualization}

The numerical data were interpreted in Gephi, an open graph visualization platform. Distance matrices generated in JPathfinder were inputted into R, a programming software used for statistical computing, to generate coordinates for each node from a Multi-Dimensional Scaling (MDS) command. This two-dimensional coordinate system was used to place each node within proximal orientation to one another according to distance from one node representing a concept from general chemistry to all other nodes in the network (Spekkink, 2015a). This was done to correspond the distances from JPathfinder to the proportional links visualized in Gephi. These dimensions were inserted into an edge table that also indexed each node as a stimulus word and defined links made between stimulus words according to their respective proximity data. Each network was transformed using a combination of Network Splitter 3D, Multi-Dimensional Scalar (MDS) Statistics algorithm, and MDS layout as tools for data analysis (Barão, 2014; Spekkink, 2015b). Together, the layouts organized the networks into clusters of closely related topics while maintaining the nodes' correct orientation in network space.

\section{RESULTS AND DISCUSSION}

\section{Knowledge Structures}

The MDS and Network Splitter layouts transformed the data into a three-dimensional space that clustered the most related concepts while maintaining the correct orientation of the stimuli, now referred to as nodes, in the Gephi visualization. Note that although each figure is obtained in three dimensions, all their features cannot be observed in this manuscript.

A concept's placement in this visual space is dependent on its favored relatedness to a group of nodes over others present in the knowledge structure, and the subsequent connections that the nodes within that group make to form the entirety of the structure. The closer a node is pulled in toward a group, it becomes part of a cluster of nodes that a student considers having a common, thematic value. Thus, the second comparison that can be made between groups lies in the tight connections formed within the two main clusters shared between them.

An important implication to note: central concepts cannot directly be determined visually from the structures shown. The central concept for each knowledge structure, identified via eccentricity values in JPathfinder, does not lie at the geometric center of the structure. These structures, furthermore, maintain the distances reflected by each link established. If a central concept were pushed into the direct center, the relatedness between each concept to one another would be skewed from distortion of the distances. Therefore, these visualizations created in Gephi can only be used to examine the clusters and associations between concepts through spatial distance and layout options available in the program. As a result, the physical "center" of the knowledge structure will be referred to as the "middle". This is to distinguish from the true central concept-this will be discussed in the section titled "Eccentricity."

Chemistry Background. Structures for both the high- and low-scoring chemistry students are marked similarly in overall appearance at first glance. For both chemistry placement exam knowledge structures (Figures 1 and 2), 


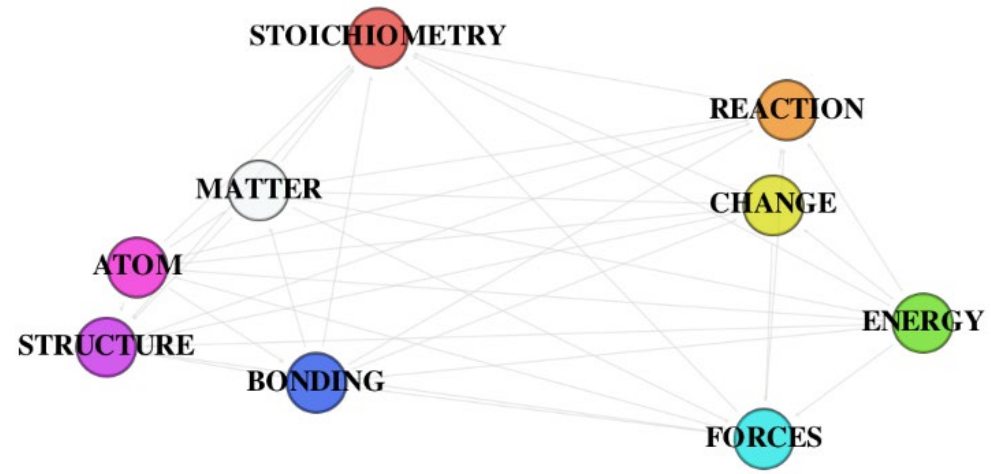

Figure 1. High- scoring chemistry students' knowledge structure

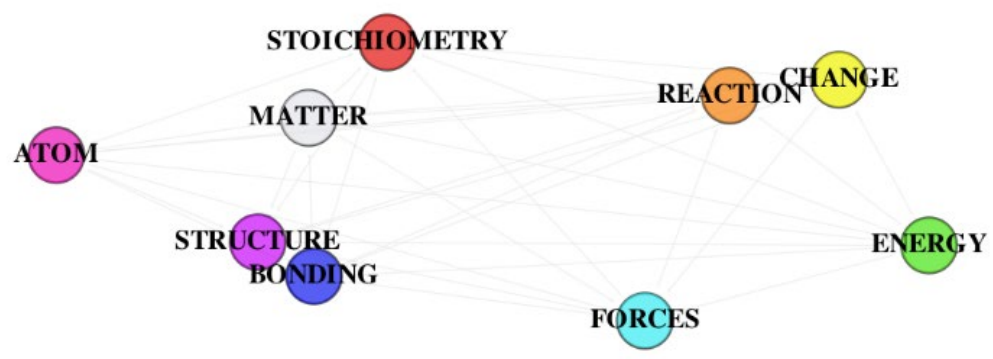

Figure 2. Low- scoring chemistry students' knowledge structure

there is a clear separation between two distinct groupings of the stimuli: the first contains stoichiometry, matter, atom, structure, and bonding whereas the second contains reaction, change, energy, and forces. The relative position of each node is maintained in both knowledge structures. Concepts such as stoichiometry, matter, bonding, and reaction maintain their placement in both groups. Yet, variations in distance between them and other neighboring concepts - which we consider as a measure of relatedness - and movement of other nodes within each structure constitute the important differences in the organization of knowledge between the high- and low-scoring students on the chemistry placement exam.

The most visible difference observed between these knowledge structures is the overall shape that constructs them. The high- scoring chemistry students' structure (Figure 1) is seemingly more compact than the low-scoring chemistry students' (Figure 2), which is diffused as the outer left and right edges-atom and forces, respectively extend further from the middle of the structure. The fact that the low-scoring students' structure is more spread out over space reveals the isolation of these two concepts as opposed to that of high-scoring students,, which associated atom more closely to the structure and matter as well as forces to energy. Yet, the high- scoring students considered stoichiometry as a markedly different separate topic, whereas the low- scoring students more strongly associated it with matter. The apparent isolation of these topics may indicate misconceptions in both groups that these fundamental ideas of general chemistry are not as related as other concepts. For example, while both groups associated stoichiometry more with matter, it was placed farther from reaction. It may be found that while students find the process of solving stoichiometric problems involving algebra to be dependent on physical quantities of matter such as atoms, moles, masses, etc. In order to achieve an answer, they miss the extended significance of the relationship between the solution of such problems and chemical reactions.

While both groups strongly associate reaction with change, there is a significant difference between how each group associates change with energy and forces. the structures in terms of the relationships between change and energy and forces. Students are not yet expected to fully understand the role that energy and forces play in chemical reactions, as intermolecular forces and energy are more emphasized in the second course of the general chemistry series at this research university. However, those with more efficient chemistry prior knowledge, that is, the high- scoring chemistry students, could distinguish stronger connections between broader themes. The low- scoring chemistry group established structure and bonding as the tightest pair out of both knowledge structures. It is also surprising to note that these students displayed a shorter distance between bonding and forces than the high-scoring students. This could explain why forces is more drawn to the middle of the structure, increasing its distance from energy: it is associated equally with both. While the significance of this relationship is obvious to chemistry experts, the low- 
scoring students do not form a cluster of the other concepts that contribute to this area of chemistry-atom, matter, structure, and bonding - in the way that the high-scoring students do. The way bonding is taught in the first course of this series also influences how students think of it: it is represented by ball- and- stick models, which give the impression that a bond is a physical entity instead of something comprised of energy and held together by forces. This is reflected in bonding's placement near matter in comparison to the high- scoring students' structure. The placement of atom is also of note, as it is further removed from the rest of the concepts than in other networks and is also considered an outlier topic. In this general chemistry course, atom is introduced in terms of the scientists and experiments that contributed to its discovery, leaving students to make connections to other topics largely by themselves.

In comparison, students considered to have an effective prior knowledge of chemistry, that is, the high-scoring group, established broader connections to construct larger clusters of related concepts more so than students with a less meaningful prior understanding of chemistry, an idea supported by the constructivist theory (Ausubel et al., 1986; Richardson, 1997; Smilkstein, 1991). The tightly formed pairs in the low- scoring chemistry knowledge structure reveal an attempt to establish connections that may have aided them in taking the first course in general chemistry; however, upon completion of the course the knowledge structure lacks further association to other learned topics, such as atom to matter, structure, and bonding. Students often miss connections between the submicroscopic and the macroscopic and fail to realize the submicroscopic explanations for the macroscopic phenomena they observe (Taber, 2013), which makes connecting concepts such as these to one another and have the potential to increase difficulty in understanding as students move further into chemistry.

Mathematics Background. As opposed to the variety of contrasting elements in the chemistry placement exam structures, the mathematics placement exam high- and low- scoring groups are constructed in a highly similar fashion. The same clusters from the chemistry placement exam structures are observed in both mathematics visualizations (Figures 3 and 4). The relative distances between most concepts are also visually similar-though there are minor deviations of two concepts and in the strength of similarity measured by minute fluctuations in these distances. In the low- scoring mathematics group (Figure 4), stoichiometry maintains the same position as in the high- scoring mathematics students (Figure 3), but with the change in location of matter from the high-scoring group, it perhaps belongs to neither of the main cluster groups established but rather is singled out. The biggest difference between the two networks was placement of matter: in the high- scoring students, it is more closely associated with stoichiometry and pushed inward toward the middle of the structure to decrease its distance with bonding. In the low- scoring students, matter is very tightly associated with atom. Another small dissimilarity between mathematics placement groups is in the placement of energy within the cluster on the right. Relative to the high- scoring mathematics group, the low-scoring mathematics students decreased the distance between reaction and change.

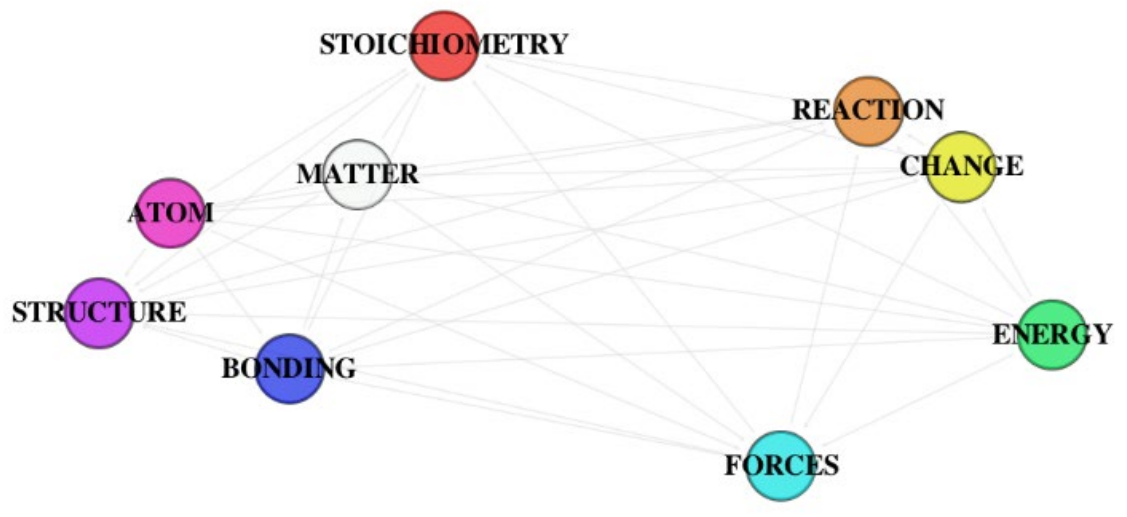

Figure 3. High- scoring mathematics students' knowledge structure 


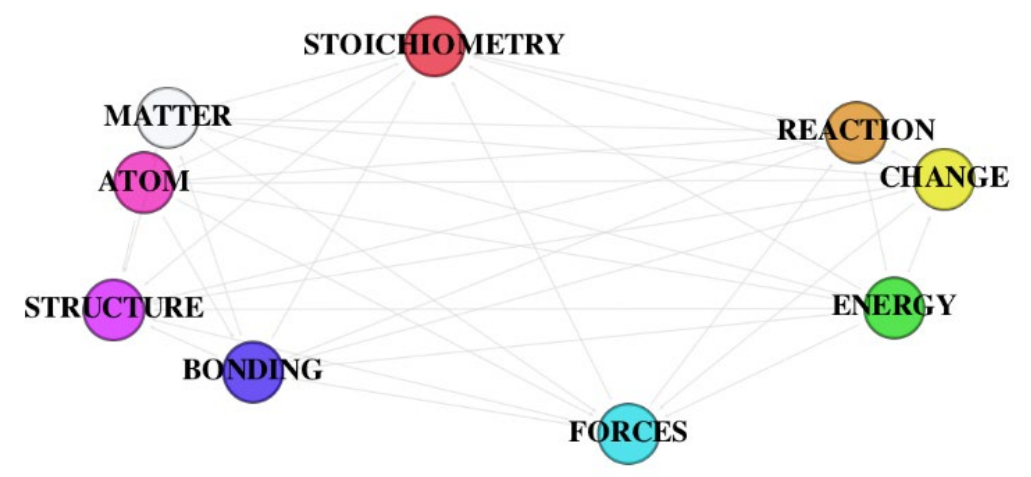

Figure 4. Low- scoring mathematics students' knowledge structure

Previous studies have shown that a firm prior understanding of stoichiometry and high mathematical literacy serve as strong predictors of a student's success in chemistry (Bird, 2010; Cambridge, 2012; Tai, Ward, \& Sadler, 2006). However, the results from the mathematics placement exam did not indicate that successful prior knowledge in mathematics distinguished students from those whose mathematics knowledge is deemed ineffective. Based on the high similarity of these knowledge structures, it cannot be confirmed that mathematics prior knowledge is a good differentiator of how students organize chemistry knowledge in their minds. Further analysis of eccentricity data is required to examine how students access the most significant information within their knowledge structure for understanding the first course in a general chemistry series.

Gender. Examination of the knowledge structures of men and women (Figures 5 and 6) participating in this study illuminated the most dramatic differences out of all the groups that were investigated. The females' knowledge structure (Figure 5) is composed of the predominant main clusters observed in all prior groups: stoichiometry, matter, atom, structure, and bonding; reaction, change, energy, and forces. The overall layout of the structure appears denser than the previously explored structures, however, and significantly smaller distances between concepts indicate high association between them: matter, atom, and structure are more tightly connected as a sub- cluster. Stoichiometry is also visibly closer to reaction, proposing a bridge between the two main clusters; the association between bonding and forces could possibly act in a similar manner.

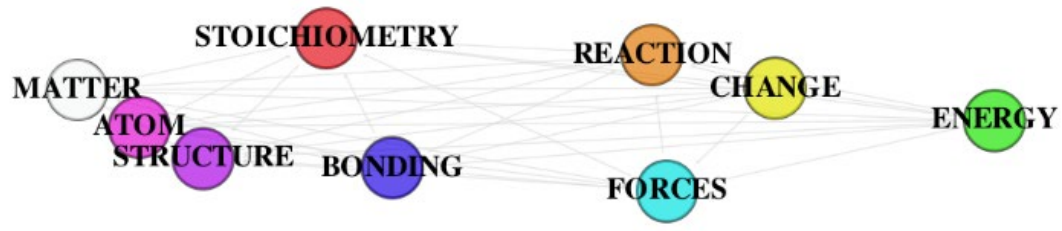

Figure 5. Female participants' knowledge structure

The male participants' knowledge structure (Figure 6) does not nearly resemble any of the previously examined groups and does not contain the well-defined main clusters seen prior. The five identified clusters for males are as follows: structure, stoichiometry, and atom; reaction and change; matter and bonding; energy and forces. A similar pairing is maintained between reaction and change, but its location has shifted upward and to the left to take the place of stoichiometry, which has gained an association between structure and atom. Atom has now moved toward the lower left of the structure and matter has moved into the middle with bonding. This is significant because no other knowledge structure organized concepts into the direct middle of the knowledge structure. Recall that this does not infer that the nodes placed in this manner are the central concept that all concepts are organized around. 


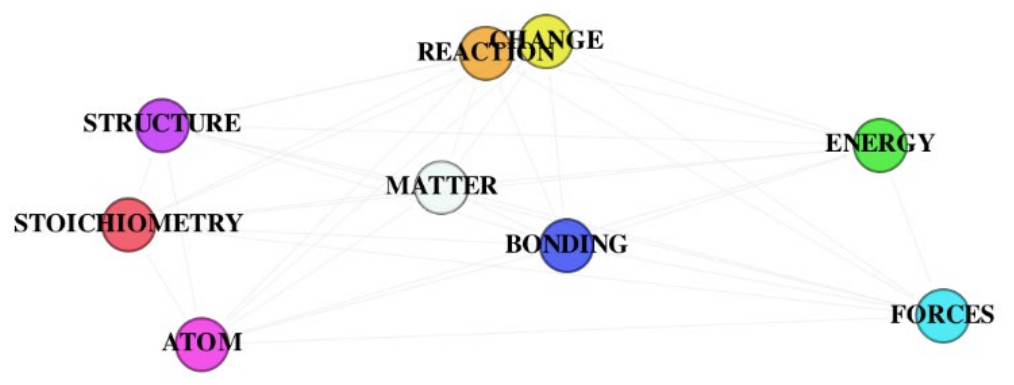

Figure 6. Male participants' knowledge structure

The placement of concepts within the males' structure is markedly different from every other in this study; male students overall are learning and organizing information in an extremely different way than female students. The formation of small clusters compared to the females' distinct polarization of two large clusters affirms the notion that males tend to exhibit left- lateralized processing in their brains in the establishment of short pathways from one node to another to generate a logical association between all concepts learned in this course using critical thinking (Bell, Willson, Wilman, Dave, \& Silverstone, 2006; Speck et al., 2000). Stronger relationships are constructed between tightly associated topics, such as reaction and change or energy and forces. The near proximity of each of these tight associations to other clusters containing two to three closely related concepts may reveal that, compared to females' organization of topics, males may gain their understanding of general chemistry through the construction of associations through critical thinking. For instance, a male's meaningful understanding of the relationship between reaction and change is useful to his ability to determine that a reaction is the result of bonding, which subsequently determines the structure of matter.

In the females' knowledge structure, organization-- a main characteristic of bilateral brain activity-- of the general chemistry course content appears to be favored over the establishment of specific connections between two concepts, thus adhering to Speck et al.'s (2000) finding that females exhibit bilateral brain activation. This approach is employed to illustrate understanding of the separate parts of the general chemistry course: lecture, where a professor demystifies the basis of chemistry as matter composed of atoms, versus laboratory, where students witness reactions and change that are typically difficult to visualize in lecture. Yet, these aspects of the general chemistry course remain separate in two distinct clusters, unlike the male participants' knowledge structure. One explanation for this polarization of two groupings, and the weak association between them, may be a result of difficulty constructing new relationships between previously learned and unfamiliar concepts or may have lacked a meaningful foundation in key concepts presented in the general chemistry course (Ausubel et al., 1986; Cavallo, 2004).

Overall. The overall knowledge structure (Figure 7) of all participating students most resembles those of the high- scoring chemistry group, both mathematics groups, and the female group (Figures 1, 3, 4, and 5).

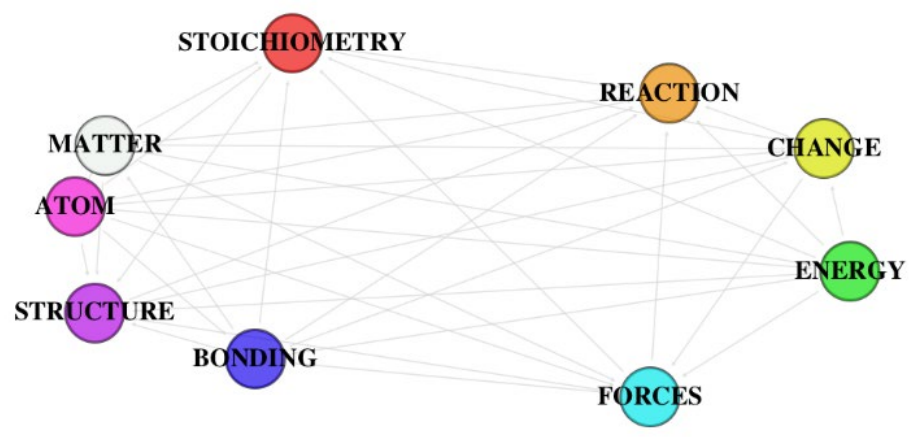

Figure 7. Overall knowledge structure

One proposed reason for this is because females composed $66.6 \%$ of the participants in the study, and so it follows that the structures for each other set of students would more strongly resemble theirs. The distance between the two concept clusters is wide, and there are strong connections between concepts within each cluster. Overall, it appears that with a firmer foundation of beginning chemistry concepts learned before this course, students should be able to create a more interconnected network of information in their future chemistry courses. 


\section{Eccentricity}

The knowledge structures visualized are representative of the strength of connections between concepts that sit most comfortably in visual space. However, we must examine centrality data through eccentricity values because the networks measure distances between nodes; they do not provide accurate representations of the physical placements of the nodes in three-dimensional space. Eccentricity, a calculation from JPathfinder, reveals the maximum number of links a node makes with other nodes in the network, which are different from the structures determined with Gephi. In Table 2, the concepts with higher eccentricity values are the ones that are more weakly connected to other concepts. Thus, the stimulus words with the lowest eccentricity values have been deemed the most central concepts in the knowledge structure (see Table 1).

Table 2. Central Concepts and Correlating Eccentricity Values ${ }^{a}$

\begin{tabular}{|c|c|c|c|c|c|c|}
\hline Nodes & C.H. & C.L. & M.H. & M.L. & F. & M. \\
\hline Atom & 6 & $3^{*}$ & 4 & 6 & 6 & 5 \\
\hline Bonding & 5 & 4 & 5 & 5 & 5 & 6 \\
\hline Energy & $4^{*}$ & 5 & 5 & $4^{*}$ & $4^{*}$ & 6 \\
\hline Matter & 7 & 4 & $3^{*}$ & 7 & 7 & 6 \\
\hline Change & 5 & 5 & 4 & 5 & 5 & 5 \\
\hline Forces & $4^{*}$ & 5 & 6 & $4^{*}$ & $4^{*}$ & 7 \\
\hline Stoichiometry & 7 & $3 *$ & 6 & 7 & 7 & $4^{*}$ \\
\hline Structure & 6 & 5 & 6 & 6 & 6 & 7 \\
\hline Reaction & 6 & 4 & 5 & 6 & 6 & $4^{*}$ \\
\hline
\end{tabular}

C.H. (Chemistry High-scoring), C.L. (Chemistry Low-scoring), M.H. (Math High-scoring), M.L. (Math Low-scoring), F. (Females), and M. (Males)

${ }^{a}$ Calculations of eccentricity and centrality were derived from JPathfinder. The highest eccentricity values denote the concepts with the largest deviations from the center of the knowledge structure. Energy, atom, forces, and stoichiometry are the central concepts

A professor who taught this course when the WAT was administered to the participants and two undergraduate researchers determined that the central concepts that students should have identified are matter, change, and atom. The proximity of the groups' central concept(s) to the known core concepts could indicate prior knowledge's role in the overall understanding of the first course in the general chemistry series as well as a measure of their success.

Chemistry Knowledge. The central concepts for the high- scoring students on the chemistry placement exam were energy and forces, whereas the low-scoring students identified atom and stoichiometry as the centers of their knowledge structure. Recall that these centers cannot be placed in the 'exact middle' of the knowledge structures. Proximity of the student- identified central concepts to other stimulus words in the knowledge structure may reveal how students access their knowledge structures for understanding. For the high- scoring students on the chemistry placement exams, the concepts that deviated the least from the center were bonding and change, each of which had an eccentricity value of 5 . Students who created these associations were predicted to have increased success in the class because of these concepts' importance to the course's curriculum. Concepts deviating most from the central concept were stoichiometry, which low-scoring students identified as a center, and matter. As for the low-scoring students, the concepts that deviated least from their centers were bonding, matter, and reaction. Matter also had closer proximity to the student's identified center, stoichiometry, than the high- scoring chemistry students. Reaction also had characteristic proximity to change, yet the emphasis of bonding as a near center may reveal their understanding of its close relationship with structure in the knowledge structure as well as their over- arching significance to the student- identified center, atom. The placement of these concepts within their knowledge structure spans in both main clusters identified earlier in this study. The low- scoring students also did not display high eccentricity values in comparison to the other five groups investigated, such as 6 or 7, but stayed in the close range of 3-5 for all stimulus words.

Mathematics Knowledge. The central concept for the high- scoring students on the mathematics placement exam was matter. This contrasts with the low- scoring students in mathematics, who, like the high- scoring chemistry students, considered energy and forces to be central. Recall that major differences were not observed between the Gephi visualizations of these high- and low- scoring students. The eccentricity data, however, illuminates a major difference between the two. Matter, with its eccentricity value of 3 , was determined to be the central concept for the mathematics high-scoring students. This coincides with matter's location within the knowledge structure: it moves toward the middle and remains closely associated with atom, another known central concept, which also demonstrated another close eccentricity value, 4, to the center. Similarly, change also had a low eccentricity value of 4 . This means that it does not deviate far from the student- identified center and thus may act like another central node on the right side of the structure. While both high- and low- scoring students establish similar connections within their minds, the way they access the heart of their learned chemistry knowledge seems to vary. This is reflected in earned placement scores, with the high- scoring students recognizing the key concepts required to understand for success in the first course. 
Although the low- scoring students also established the high- scoring students' relationships in their knowledge structure, they exhibited emphasis on the right main cluster focusing on energy and forces, both of which have eccentricity values of 4 . These concepts do pertain to one of the known central concepts, change-which has an eccentricity value of 5 - as they play a role in chemical reactions. Bonding also exhibited an eccentricity value of 5 , but its distance from the central concept atom is larger than that observed in the high- scoring students' structure. Two of the professor-determined central concepts, atom and matter, had high eccentricity values of 6 and 7 , respectively, in this group, and thus deviate more from the students' determined centers. Whereas the high- scoring students could locate the three most meaningful concepts in the first course of general chemistry, the low-scoring students identified one, indicating the presence of student misconception of the significance of this course and where this misconception lies within their knowledge structure: with topics such as bonding, atom, and, matter.

Gender. In a way comparable to their differences in the organization of their knowledge structures, females and males identified markedly contrasting central concepts. While the females considered energy and forces - both with eccentricity values of $4-$ as centers, these concepts deviated most in the males' knowledge structure. Energy and forces had eccentricity value of 6 and 7, respectively; their central concepts were stoichiometry and reaction, both had eccentricity values of 4 . Similarly, these male-identified centers deviated most in the females' knowledge structure: with stoichiometry had a value of 7, and reaction had one of 6 . Despite these contrasting observations, both groups identified the same near- center, change, a known central concept. For females, this had the same amount of deviation from the center as bonding, and for the males it was atom. Neither group also identified matter as being central in their knowledge structure.

Overall Eccentricity Data. It was found that half the groups in this study identified the same two concepts, energy and forces: high- scoring chemistry, low- scoring mathematics, and females. The low- scoring chemistry, highscoring mathematics, and males all identified varying central concepts. Of note is that low-scoring chemistry and the male participants both determined stoichiometry as being central in both their structures, although that concepts did not appear as tightly clustered as other concepts.

\section{Analysis Using the Chemistry Triplet}

The chosen stimulus words can also be categorized with regards to the chemistry triplet, and analyzing the generated knowledge structures in terms of the chemistry triplet can provide a different dimension of analysis. As stated above, the chemistry triplet considers three aspects of chemistry knowledge: the submicroscopic, the macroscopic, and the symbolic. The submicroscopic is generally thought of as anything relating to the particle level, such as the atoms that compose matter, whereas macroscopic represents the physically tangible or visible element of the triplet. Symbolic is everything that makes up the way chemistry knowledge is represented and communicated.

The first step in analyzing the knowledge structures according to the triplet was to categorize each stimulus word as one or more parts of the triplet. Two undergraduate students in chemistry and a professor of general chemistry did this by using a method that aimed to mimic how the students taking general chemistry perceived these concepts. This was conducted by looking at the list of top responses that students gave for each stimulus and trying to categorize them based on the chemistry triplet. The categorization of each stimulus word was chosen to most accurately reflect how entry-level general chemistry students viewed each topic (Table 3). The top response words for each stimulus shown below were also taken into account.

Table 3. Categorizations of Stimulus Words According to the Chemistry Triplet

\begin{tabular}{cc}
\hline Stimulus Word & Categorization \\
\hline Atom & Submicroscopic \\
\hline Bonding & Submicroscopic \\
\hline Energy & Macroscopic \\
\hline Matter & Macroscopic \\
\hline Stoichiometry & Symbolic \\
\hline Forces & Macroscopic, submicroscopic \\
\hline Change & Macroscopic, symbolic \\
\hline Structure & Submicroscopic, symbolic \\
\hline Reaction & Submicroscopic, symbolic \\
\hline
\end{tabular}

The stimulus words categorized solely as submicroscopic were atom and bonding. The top responses for both stimulus words were largely identified as submicroscopic and dealt with topics on the atomic and subatomic levels. In the case of bonding, this could have been due to the way it was taught: as a force between atoms or molecules.

The macroscopic concepts included matter and energy. The response words for matter primarily concerned visible phenomena, while it was deemed that the responses for energy reflected an understanding of energy that 
was not yet viewed through the submicroscopic lens. The responses listed are simpler to view on a larger scale and do not reveal that the students necessarily understood what energy appears as at the subatomic level.

Stoichiometry was the only stimulus word categorized solely as symbolic. In the general chemistry course, the emphasis is placed on stoichiometry as a means of converting between units. Few responses reference anything conceptual, either macroscopic or submicroscopic; this lack of conceptual understanding could explain stoichiometry's isolated placement from the other stimulus words on most of the generated networks.

However, the students appeared to think of the other stimulus words in terms of more than one level of understanding, although no stimulus could be clearly classified as all three.

Forces was categorized as both macroscopic and submicroscopic. Many of its response words related primarily to a basic knowledge of physics, one whose macroscopic effects are taught at the basic level. However, the "bonding" and "intermolecular" responses appeared to indicate that students are beginning to understand forces at the submicroscopic level as well. These response words also relate primarily to chemistry, indicating that students are developing a more nuanced understanding of forces as both a physical and chemical phenomenon.

Change was considered to be both macroscopic and symbolic. In this general chemistry course, students are taught about two primary types of change-physical and chemical-both of appear on the response words for change. The course also taught that indications of a chemical change can include a change in color or phase, both are also present on the list of response words. These are simple to think of in terms of what a student might physically see in the lab without taking into account what happens at the atomic level. However, the presence of "delta" and to some extension "reaction" on this list could be evidence that students are also thinking of how change is represented symbolically, especially in chemical problems.

Structure and reaction were both classified as submicroscopic as well as symbolic. The majority of the response words for structure concerned goings-on at the submicroscopic level. However, "diagram" is indicative of the symbolic and the fact that this course emphasized symbolic models such as Lewis structures.

Reaction was classified as submicroscopic and symbolic due to a few key response words. The majority of them appear to refer to submicroscopic aspects of reactions, but the presence of "balance", "product", and "reactant" also led to its classification as symbolic. Balancing reactions on paper is heavily emphasized in this course, as is being able to identify the products and reactants when given a reaction on paper.

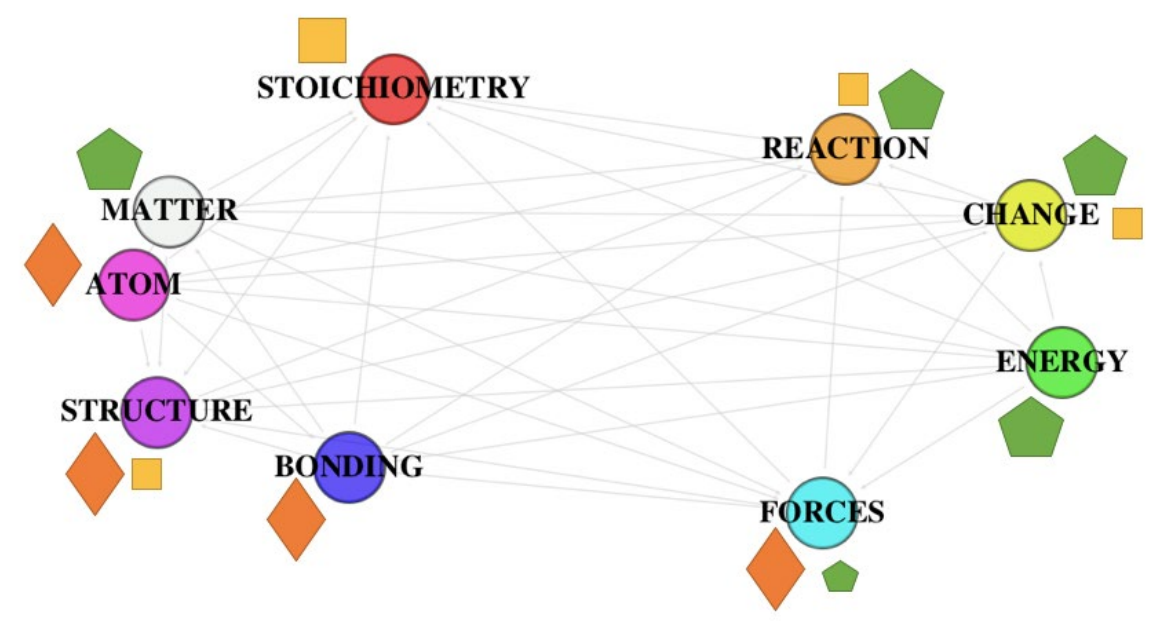

Figure 8. Overall knowledge structure for the first course in General Chemistry. Each stimulus word has been classified and marked as a part of the chemistry triplet to add another layer of understanding to the network. Here, the orange diamond represents submicroscopic, the yellow square represents symbolic, and the green pentagon represents macroscopic

Looking at the overall knowledge structure (Figure 8) with regards to the chemistry triplet does not reveal a concrete pattern to student thinking. However, many of the submicroscopic words are gathered on the left side of the graph, while the right side consists of most of the macroscopic-identified terms. It is also interesting to note there is no one region of the graph occupied mostly by the topics classified as symbolic; those are spread all around the graph and have no main region where they are concentrated. Within the macroscopic topics, no such associations exist for the symbolic terms, which might be a lack of a general understanding for the symbolic topics that exists for the other two categories. This could also be a result of the way the course is taught, with conceptual understanding being more highly emphasized for most topics. As such, the lack of patterning with the symbolic terms can be understood to partially be a product of the way the course was taught and the topics that were 
emphasized. As stated before, stoichiometry, the only stimulus identified solely as symbolic, is not a part of any one cluster. This contrasts with the other stimuli, all are a part of two main clusters.

It is not easy to discern a constant pattern to this knowledge structure in terms of the chemistry triplet, but it has some obvious influence on how students think and associate the chemical terms.

\section{LIMITATIONS}

Although the WAT does not constitute all chemistry knowledge in a student's structure, it is a good method to determine where students' misconceptions exist. The importance of relationships between concepts may differ according to professors, causing these visualizations not to be representative of all general chemistry courses. A certain degree of consistency and generalization is required for qualitative analysis. For this reason, the grouping of responses is an aspect in which the data may not accurately reflect a more widespread population.

Along the same lines, without the knowledge structures of experts as a comparison, there is no way to know what the ideal structure looks like. Without expert comparison, student understanding of each topic cannot necessarily be enhanced to the greatest degree.

The cut-offs between what is considered a high- or low-scoring student could also be improved. Initially, whether a student had taken Advanced Placement (AP) Chemistry was going to be used as a measure of their prior knowledge in chemistry. Advanced Placement classes are offered in the United States as high school courses that are intended to go above and beyond regular classes and are associated with more rigorous workloads and more in-depth material. However, only 102 of the responding students $(16.6 \%)$ had taken AP Chemistry. This was not a large enough portion of the population to create visuals that would accurately reflect differences in the general knowledge of those who had taken AP Chemistry and those who had not. Also, the WAT was administered shortly before students had completed their general chemistry course fully. In the future, grades may be useful as better predictors of success in comparison with the population of students in each knowledge structure group.

Most of the participants in the study were female (68\% vs. $32 \%)$. The large imbalance is an important factor to keep in mind while reading the analysis highlighting the differences between the knowledge structures of Highand Low-Achieving students in both mathematics and chemistry. It is difficult to discern the exact interplay between the role of gender and prior knowledge. This limitation should be acknowledged, and further studies should aim to recruit the same number of students from each gender group to distill the role of each factor on the knowledge structures.

Additionally, the visualizations represented by Gephi are limited and cannot fully represent the data collected due to the codes and processing platforms currently available for use. Although this program cannot represent the students' knowledge structures with complete accuracy, Gephi can visually represent the clustering and organization within students' minds and the close connections between these concepts.

\section{IMPLICATIONS}

Knowledge structures are one of many reference points that can shed light on students' comprehension of the material and track their progress throughout their subsequent chemistry courses. This method could be of use to instructors, both high school and university- level, in determining connections students make between concepts and which concepts cause confusion and to analyze how students' organization of concepts changes throughout time and as they progress through their education. Students can also make personal use of this method by examining a representation of the structure that best fits their demographic to pinpoint their own misconceptions of material they are expected to understand. As a result, they can focus their studies on finding common factors that intersect with the most important concepts in their current courses. However, it must be noted that the WAT as a method fails in creating an individuals' knowledge structure because it is not a good representation of the most significant regions of their learning. For a WAT that has a large group of participants, extraneous subjects that might not be used in problem- solving are able to be eliminated and the broader themes are highlighted. In addition, researchers may implement the WAT due to its effectiveness in achieving a wide variety of data in an efficient manner.

\section{CONCLUSIONS}

Future research will include an examination of both high school students' and experts' knowledge structures. These structures will not only serve as comparative tools for the ideal organization of knowledge in an individual's mind; they will also be used to track the evolution of one's knowledge structure as more learned knowledge is acquired over time. Additionally, a WAT will be developed to identify and compare the effects of a constructivist approach to teaching and a traditional approach on students' knowledge structures. 
The chemistry placement exam data revealed a variety of differences in organization and strength of connections within the knowledge structures of both sides of the scoring spectrum for this exam. However, the mathematics placement data did not entirely illuminate what separates "successful" students from their counterparts solely based on the visualization of their knowledge structures due to the high similarity of each group's construction of the relationships between concepts. The way students access these structures in their minds is highlighted via eccentricity, and perhaps problem- solving would be a more viable tool to see how students successful in mathematics perform in their chemistry courses compared to those with insufficient mathematics knowledge. Success of both high- scoring chemistry and mathematics students cannot be confirmed without comparison to the final grades earned in this general chemistry course.

In comparison to the similarities observed in the categorization of students based on placement exam score, the separation of genders proved fruitful in highlighting the contrasting methods of organization of concepts within females' and males' knowledge structures. In addition, their contrasting identifications of central concepts also prove that females and males are interpreting a different significance from the first course in a general chemistry series. Thus, this visible gap between the student population's understanding must be addressed.

\section{REFERENCES}

Ashcraft, M. H. (1978). Property norms for typical and atypical items from 17 categories: A description and discussion. Memory \& Cognition, 6(3), 227-232. https:/ / doi.org/10.3758/BF03197450

Ausubel, D., Novak, J., \& Hanesian, H. (1986). Educational psychology: A cognitive view (2 ed.). New York: Holt, Rinehart and Winston.

Bahar, M., \& Hansell, M. H. (2000). The relationship between some psychological factors and their effect on the performance of grid questions and word association tests. Educational Psychology, 20(3), 349-364. https:/ / doi.org/10.1080/713663739

Bahar, M., Johnstone, A. H., \& Sutcliffe, R. G. (1999). Investigation of students' cognitive structure in elementary genetics through word association tests. Journal of Biological Education, 33(3), 134-141. https:/ / doi.org/10.1080/00219266.1999.9655653

Baker, O. (1993). How constructivist theory and research inform educational policy. Paper presented at the Annual Meeting of the American Educational Research Association, Atlanta, Georgia.

Barão, A. (2014). Gephi plugin: Network Splitter 3D.

Bell, E. C., Willson, M. C., Wilman, A. H., Dave, S., \& Silverstone, P. H. (2006). Males and females differ in brain activation during cognitive tasks. NeuroImage, 30(2), 529-538. https:/ / doi.org/10.1016/j.neuroimage.2005.09.049

Bird, L. (2010). Logical reasoning ability and student performance in general chemistry. Journal of Chemical Education, 87(5), 541-546. https://doi.org/10.1021/ed8001754

Bodner, G. M. (1986). Constructivism: A theory of knowledge. Journal of Chemical Education, 63(10). https://doi.org/10.1021/ed063p873

Cambridge, D. (2012). The relationship between student's quantitative skills, application of math, science courses, and science marks at single-sex independent high schools. (Ed.D.), St. Joseph's University, Ann Arbor, United States. Retrieved from https://eric.ed.gov/? ProQuest Dissertations \& Theses A\&I database.

Cavallo, A. M. J. P., Wendell H.; Rozman, Michelle. (2004). Gender differences in learning constructs, shifts in learning constructs, and their relationship to course achievement in a structured inquiry, yearlong college physics course for life science majors. School Science and Mathematics, 104(6). https:/ / doi.org/10.1111/j.19498594.2004.tb18000.x

Clauser, B. E. (2007). The life and labors of Francis Galton: A review of four recent books about the father of behavioral statistics. [Francis Galton: Pioneer of Heredity and Biometry, M. Bulmer; Extreme Measures: The Dark Visions and Bright Ideas of Francis Galton, M. Brookes; The Biographer's Tale, A. S. Byatt; A Life of Sir Francis Galton: From African Exploration to the Birth of Eugenics, N. W. Gillham]. Journal of Educational and Behavioral Statistics, 32(4), 440-444. https:/ / doi.org/10.3102/1076998607307449

Forster, P. (1999). Applying constructivist theory to practice in a technology-based learning environment. Mathematics Education Research Journal, 11(2), 81-93. https:/ / doi.org/10.1007/BF03217062

Garskof, B. E., \& Houston, J. P. (1963). Measurement of verbal relatedness: An idiographic approach. Psychological Review, 70(3), 277. https:/ / doi.org/10.1037/h0041879

Gulacar, O., \& Bowman, C. R. (2014). Determining what our students need most: exploring student perceptions and comparing difficulty ratings of students and faculty. Chemistry Education Research and Practice, 15(4), 587-593. https:/ / doi.org/10.1039/C4RP00055B 
Gulacar, O., Sinan, O., Bowman, C. R., \& Yildirim, Y. (2015). Exploring the changes in students' understanding of the scientific method using word associations. Research in Science Education, 45(5), 717-726. https:/ / doi.org/10.1007/s11165-014-9443-9

Hoffmann, R., \& Laszlo, P. (1991). Representation in chemistry. Angewandte Chemie, 30(1), 1-16. https:// doi.org/10.1002/anie.199100013

Johnstone, A. H. (1982). Macro- and microchemistry. School Science Review, 64, 377-379.

Johnstone, A. H. (2006). Chemical education research in Glasgow in persepctive. Chemistry Education Research and Practice, 7(2), 49-63. https:// doi.org/10.1039/B5RP90021B

Jung, C. G. (1910). The association method. American Journal of Psychology, 21(2), $219-269$. https:/ / doi.org/10.2307/1413002

Kempa, R., \& Nicholls, C. E. (1983). Problem-solving ability and cognitive structure-an exploratory investigation. European Journal of Science Education, 5(2), 171-184. https:/ / doi.org/10.1080/0140528830050205

Kilner, W. C. (2014). The Chem-Math Project: Enhancing Success in General Chemistry through the Integration of Mathematics, Problem-Solving and Conceptual Understanding. An Action-Research Study. Retrieved from ERIC database https: / / search.proquest.com/ docview /1720061323?accountid=14505

Kostova, Z. R., Blagovesta. (2008). Word association test for studying conceptual structures of teachers and students. Bulgarian Journal of Science and Education Policy, 2(2), 209-231.

Kounios, J., \& Holcomb, P. J. (1992). Structure and process in semantic memory: Evidence from event-related brain potentials and reaction times. Journal of Experimental Psychology, 121(4), 459-479. https:// doi.org/10.1037/0096-3445.121.4.459

Kurt, H., Ekici, G., Aktas, M., \& Aksu, O. (2013). Determining biology student teachers' cognitive structure on the concept of "diffusion" through the free word-association test and the drawing-writing technique. International Education Studies, 6(9). https:// doi.org/10.5539/ies.v6n9p187

Lee, K.-W. L. (1985). Cognitive variables in problem solving in chemistry. Research in Science Education, 15(1), 43-50. https:/ / doi.org/10.1007/BF02356524

Lee, K.-W. L., \& Fensham, P. J. (1996). A general strategy for solving high school electrochemistry problems. International Journal of Science Education, 18(5), 543-555. https:/ / doi.org/10.1080/0950069960180504

Lee, K.-W. L., Goh, N. K., Chia, L. S., \& Chin, C. (1996). Cognitive variables in problem solving in chemistry: A revisited study. Science Education, 80(6), 691-710. https://doi.org/10.1002/(SICI)1098237X(199611)80:6<691::AID-SCE4>3.0.CO;2-E

Murphy, K., Holme, T., Zenisky, A., Caruthers, H., \& Knaus, K. (2012). Building the ACS exams anchoring concept content map for undergraduate chemistry. Journal of Chemical Education, 89(6), 715-720. https:// doi.org/10.1021/ed300049w

Nakiboglu, C. (2008). Using word associations for assessing non major science students' knowledge structure before and after general chemistry instruction: the case of atomic structure. Chemistry Education Research and Practice, 9(4), 309-322. https:/ / doi.org/10.1039/b818466f

Noss, R., \& Hoyles, C. (2017). Constructionism and Microworlds. In E. Duval, M. Sharples, \& R. Sutherland (Eds.), Technology Enhanced Learning: Research Themes (pp. 29-35). Cham: Springer International Publishing.

Petrucci, R. H., Herring, F. G., Madura, J. D., \& Bissonnette, C. (2011). General chemistry: Principles and modern applications (10th ed.). Toronto: Pearson.

Pinarbasi, T., \& Canpolat, N. (2003). Students' understanding of solution chemistry concepts. Journal of Chemical Education, 80(11), 1328-1332. https:/ / doi.org/10.1021/ed080p1328

Richardson, V. (1997). Constructivist teacher education: Building a world of new understandings. London: Routledge/Falmer.

Rixse, J. S., \& Pickering, M. (1985). Freshman chemistry as a predictor of future academic success. Journal of Chemical Education, 62(4), 313. https:/ / doi.org/10.1021/ed062p313

Schvaneveldt, R. W. (1990). Proximities, networks, and schemata. In R. W. Schvaneveldt (Ed.), Pathfinder associative networks: Studies in knowledge organization (pp. 135-148). Norwood, NJ: Ablex.

Shavelson, R. J. (1972). Some aspects of the correspondence between content structure and cognitive structure in physics instruction. Journal of Educational Psychology, 63(3), 225-234. https://doi.org/10.1037/h0032652

Shibley Jr, I. A., Milakofsky, L. M., Bender, D. S., \& Patterson, H. O. (2003). College chemistry and Piaget: An analysis of gender difference, cognitive abilities, and achievement measures seventeen years apart. Journal of Chemical Education, 80(5). https:// doi.org/10.1021/ed080p569 
Smilkstein, R. (1991). A natural teaching method based on learning theory. Gamut, 12-15.

Speck, O., Ernst, T., Braun, J., Koch, C., Miller, E., \& Chang, L. (2000). Gender differences in the functional organization of the brain for working memory. NeuroReport, 11(11), 2581-2585. https:/ / doi.org/10.1097/00001756-200008030-00046

Spekkink, W. (2015a). MDS Layout.

Spekkink, W. (2015b). MDS Statistics

Taber, K. S. (2013). Revisiting the chemistry triplet: drawing upon the nature of chemical knowledge and the psychology of learning to inform chemistry education. Chemistry Education Research and Practice, 14(2), 156168. https://doi.org/10.1039/c3rp00012e

Tai, R. H., Ward, B. R., \& Sadler, P. M. (2006). High school chemistry content background of introductory college chemistry students and its association with college chemistry grades. Journal of Chemical Education, 83(11), 1703-1711. https:// doi.org/10.1021/ed083p1703

Talanquer, V. (2011). Macro, submicro, and symbolic: The many faces of the chemistry "triplet". International Journal of Science Education, 33(2), 179-195. https:/ / doi.org/10.1080/09500690903386435

Von Glasersfeld, E. (1990). An exposition of constructivism: Why some like it radical. In R. B. Davis, C. A. Mayer, \& N. Noddings (Eds.), Constructivist view on the teaching and learning of mathematics (pp. 19-29). Reston, VA: National Council of Teachers of Mathematics.

Wagner, W., Valencia, J., \& Elejabarrieta, F. (1996). Relevance, discourse and the 'hot' stable core social representations - A structural analysis of word associations. British Journal of Social Psychology, 35(3), 331351. https://doi.org/10.1111/j.2044-8309.1996.tb01101.x

Yücel, E. Ö., \& Özkan, M. (2015). Determination of secondary school students' cognitive structure, and misconception in ecological concepts through word association test. Educational Research and Reviews, 10(5), 660. https:/ / doi.org/10.5897/ERR2014.2022

\section{http://www.ejmste.com}

